\title{
DISCUSSION AFTER SESSION A
}

Murray: A fundamental catalogue can contain many sorts of stars for many purposes. It should include sufficient stars at an optimum magnitude for calibration of the overlapping plate surveys. I would suggest that perhaps 3000 stars in a very narrow magnitude range would be sufficient.

Fricke: I hope that we will be able to provide at least 2000 stars in the FK5 within the magnitude range 7.5 to 9 visual, and there will be others fainter than visual magnitude 7.5 in the FK5 Supplement. I am, however, not able at present to make an estimate of the total number of objects fainter than 7.5 in the FK5 Sup.

Lacroute: It is important that Heidelberg provides a large number of fundamental and supplementary stars for the photographic reduction even if the precision is not as good as for the fundamental stars brighter than visual magnitude 7.5.

Bok: (1) Obviously we must urge radio astronomers to do their utmost to add to the list of true radio stars, such as $\beta$ Persei. Such stars - once known - should be observed assiduously and be included in future fundamental catalogues.

(2) We have heard much about the importance of precise radio positions for future fundamental positions. There is a risk that from the start we shall neglect once again the southern hemisphere. We must do everything in our power to urge radio astronomers to assure that from the start there will be a proper balance between objects at northern declinations and those in the deep south.

Fricke: I suggest that the Resolutions Committee of this Symposium takes into account Dr Bok's remarks in drafting two resolutions.

Robertson (D.S.): In reply to Dr Bok's urging southern hemisphere positional radio astronomy, his words are music to my ears. The three radio telescopes that have been used for this work in the southern hemisphere are Tidbinbilla near Canberra, Island Lagoon, near Woomera and Johannesburg. Island Lagoon has been closed and offered to Australian radio astronomers by NASA. Australian radio astronomers do not appear to want it and it is likely to be demolished. Next year Johannesburg is to be closed and further work will not be possible.

Elsmore: Concerning the choice of radio sources for inclusion in a fundamental catalogue, a working party has been set up in Commission $\mathbf{4 0}$ to provide a list of calibration sources for positional work; this list will include sources for which there are no optical counterparts. Co-operation with Commission $\mathbf{4 0}$ is therefore advised.

Fricke: Such co-operation would be much welcomed by Commission 8 and, in particular, by my colleagues at Heidelberg and myself.

Dieckvoss: It may be mentioned that at Bergedorf for the declination zones north of $+40^{\circ}$ the data of the astrographic catalogue were combined with AGK2 and AGK3 to give proper motions with mean errors of \pm 0.4 per century. 